\title{
Classical Linear Stability Analysis of Energy Based Internally Heated Distributions on Bénard Porous Convection in a Micropolar Fluid Layer
}

\author{
A. Pavithra, C. E. Nanjundappa* \\ Department of Mathematics, Dr. Ambedkar Institute of Technology, Bengaluru, India \\ Email: pavithragowda1601@gmail.com, *nanjundappace@gmail.com
}

\begin{abstract}
How to cite this paper: Pavithra, A. and Nanjundappa, C.E. (2022) Classical Linear Stability Analysis of Energy Based Internally Heated Distributions on Bénard Porous Convection in a Micropolar Fluid Layer. Journal of Electromagnetic Analysis and Applications, 14, 1-18.

https://doi.org/10.4236/jemaa.2022.141001
\end{abstract}

Received: December 31, 2021

Accepted: January 25, 2022

Published: January 28, 2022

Copyright $\odot 2022$ by author(s) and Scientific Research Publishing Inc. This work is licensed under the Creative Commons Attribution International License (CC BY 4.0).

http://creativecommons.org/licenses/by/4.0/ (c) (i) Open Access

\begin{abstract}
The theoretical and numerical analysis is carried out on the effect of three types of configurations of Rayleigh-Bénard (RB) convection driven by the boundary combinations of Rigid-Rigid (R-R), Rigid-Free (R-F) and Free-Free (F-F). The $\mathrm{RB}$ convection models are distinguished by the three different temperature boundary conditions like: 1) RB1: lower and upper at fixed-temperature, 2) RB2: lower and upper with fixed-heat flux, or perfectly insulating and 3) RB3: bottom surface is fixed-temperature and top surface is fixed-heat flux. A Galerkin-type is based on the weighted residual method (WRM) which has been used to obtain the eigenvalue for gravity thermal Rayleigh number. It is noted that the porous medium of Darcy parameter $D a$ and spin diffusion (couple stress) parameter $N_{3}$ is to hasten coupling parameter $N_{1}$ and micropolar heat conduction parameter $N_{5}$ is to delay the onset of convection. Further, increase in the value of $N_{1}, N_{5}, \Lambda$ and as well as decrease in $N_{3}$ is to diminish the size of convection cells.
\end{abstract}

\section{Keywords}

Porous Medium, Galerkin Method, Micropolar Heat Conduction Parameter, Internal Heat Source, Fixed-Heat Flux

\section{Introduction}

The model of a micropolar fluid develops from the fluid flows that include rotating micro-constituents (Eringen [1]). However, much less work has been done on convection in non-Newtonian fluids such as the micropolar fluids. The 
theory of micropolar fluids, as developed by Eringen [2], has been a field of sprightly research for the last few decades especially in many industrially important fluids like paints, polymeric suspensions, colloidal fluids, and also in physiological fluids such as normal human blood and synovial fluids.

The Navier-Stokes equations cannot passably explain the motion of such fluids. Many researchers (Lebon and Perez [3], Payne and Straughan [4], Idris et al. [5], Mahmud et al. [6], Sharma and Kumar [7]) have been rigorously investigated the Rayleigh-Bénard situation in Eringen's micropolar non-magnetic fluids. From all these studies, they mainly found that stationary convection is the preferred mode for heating from below. Zahn and Greer [8] have analyzed ferrofluid pumping in a planar duct driven by spatially non-uniform traveling wave magnetic fields. This work extends recent similar analysis which examined the change in effective ferrofluid viscosity under alternating magnetic field. The governing linear and angular momentum conservation equations are numerically integrated to solve for flow and spin velocity distributions. Ruraiah et al. [9] studied analytically the onset of non-uniform temperature gradients on thermo magnetic convection in a horizontal layer of ferromagnetic confined between the rigid boundaries. Abraham [10] has investigated the problem of Rayleigh-Bénard convection in a micropolar ferromagnetic fluid layer permeated by a uniform magnetic field for stress-free boundaries. The influence of the various micropolar and magnetization parameters on the onset of stationary convection mode has been analyzed by applying the single term Galerkin method. Sunil et al. [11] studied the linear stability analysis for a micropolar ferrofluid layer, heated from below subjected to a transverse uniform magnetic field in the presence of uniform vertical rotation. For a layer, two free-flat fluid boundaries, an exact solution is obtained using normal mode analysis method. The critical magnetic thermal Rayleigh number for the onset of instability is also determined numerically by Raleigh Ritz method. Nanjundappa et al. [12] have investigated the onset of ferromagnetic convection in a micropolar ferromagnetic fluid layer heated from below in the presence of a uniform applied vertical magnetic field. The rigid-isothermal boundaries of the fluid layer are considered to be either paramagnetic or ferromagnetic and the eigenvalue problem is solved numerically using the higher order-Galerkin method.

The practical problems cited above require a mechanism to control thermomagnetic convection. One of the mechanisms to control (suppress or augment) convection is by maintaining a non-uniform temperature gradient across the layer of ferrofluid. Such a temperature gradient may arise due to 1) uniform distribution of heat sources 2) transient heating or cooling at a boundary, 3) temperature modulation at the boundaries and so on. Works have been carried out in this direction but it is still in much-to-be desired state. Rudraiah and Sekhar [13] have investigated convection in a ferrofluid layer in the presence of uniform internal heat source using the method of Ralyleigh Ritz method. The effect of non-uniform basic temperature gradients on the onset of ferroconvection has 
been analyzed (Shivakumara et al. [14], and Shivakumara and Nanjundappa [15] [16]). The critical eigenvalue solutions are obtained numerically by the method of higher order Galerkin procedure under weighted residual technique. Singh and Bajaj [17] have studied thermal convection of ferrofluids with boundary temperatures modulated sinusoidally about some reference value. The gravity-free limit is also evaluated as a function of the magnetic susceptibility, under modulation by carry out this extensive study is utilized by classical Floquet theory. Nanjundappa et al. [18] studied the effect of internal heat generation on the criterion for the onset of convection in a horizontal ferrofluid saturated porous layer in the presence of a uniform magnetic field using the Brinkman-Lapwood extended Darcy flow model with fluid viscosity different from effective viscosity. Nanjundappa et al. [19] have explored a model for penetrative ferroconvection via internal heat generation in a ferrofluid saturated porous layer. The Brinkman-Lapwood extended Darcy equation with fluid viscosity different from effective viscosity is applied to describe the flow in the porous medium. Nanjundappa et al. [20] investigated the onset of penetrative Benard-Marangoni convection in a horizontal ferromagnetic fluid layer in the presence of a uniform vertical magnetic field via an internal heating model by performing the linear stability analysis. The eigenvalue problem is solved numerically using the Galerkin type of weighted residual technique by considering either the Rayleigh number or the Marangoni number as the eigenvalue. Rômulo et al. [21] investigated the effects of thermal and turbulent on forced convection in a heated square cylinder for Two-Dimensional Simulation of the Navier-Stokes Equations. The purpose of this work is to use the Immersed Boundary Method (IBM) coupled with the Virtual Physical Model (VPM) to investigate incompressible two-dimensional Newtonian flow around a heated square cylinder at constant temperature on its surface with forced convection and turbulence. Rashad et al. [22] reported the analytical solution for convective flow of micropolar-nanofluid past of horizontal circular cylinder in a saturating porous medium. Non-similar variables are used to make the governing equations dimensionless. The local similar and non-similar solutions are obtained by using the Runge-Kutta-Fehlberg method of seventh order. Recently, Mohammad et al. [23] investigated the unsteady electromagnetic free convection flows of two-dimensional micropolar fluid through in a porous medium parallel to a vertical porous plate. This analysis has been used to transform the governing equations into its non-dimensional form by using the explicit finite difference method to obtain numerical solutions.

The theoretical and numerical analysis is carried out on the effect of three types of configurations of Rayleigh-Bénard (RB) convection driven by the velocity boundary combinations of R-R, R-F and F-F with three types of temperature perturbations akin to lower and upper at fixed-temperature, lower and upper with fixed-heat flux, or perfectly insulating and bottom surface is fixed-temperature and top surface is fixed-heat flux. Such investigation helps in 
understanding the control of thermal convection in a micropolar fluid saturated porous medium due to buoyancy force in the presence of uniform internal heat source, which has important applications in micropolar technology. The problem using a Galerkin-type of weighted residual method is solved numerically in order to determine the eigenvalues. Finally, results are shown graphically.

\section{Mathematical Formulation}

Consider an incompressible micropolar fluid-saturated Brinkman porous layer. A co-ordinate system $O X, O Y, O Z$ is chosen, $O Z$ having perpendicularly upward directions and $O X, O Y$ in the horizontal plane (Figure 1). The fluid is considered to be confined between the surfaces, which are kept at constant temperatures:

$$
T=T_{0}(z=0) \text { and } T=T_{1}(z=d)
$$

It is assumed that temperature heat source term, $Q$, at a constant rate throughout the volume of the fluid. The gravity acts perpendicularly downward directions $(\vec{g}=-g \hat{k})$.

Under assumptions of Boussinesq approximations, the equations governing the motion of a saturated porous medium with micropolar fluids:

$$
\begin{gathered}
\nabla \cdot \vec{q}=0 \\
\frac{\rho_{0}}{\varepsilon}\left[\frac{\partial}{\partial t}+\frac{1}{\varepsilon}(\vec{q} \cdot \nabla)\right] \vec{q}=-\nabla p+\rho_{0} \vec{g}-\frac{1}{k_{1}}(\xi+\eta) \vec{q}+2 \xi(\nabla \times \vec{\omega}) \\
\rho_{0} I\left[\frac{\partial}{\partial t}+\frac{1}{\varepsilon}(\vec{q} \cdot \nabla)\right] \vec{\omega}=2 \xi\left[\frac{1}{\varepsilon}(\nabla \times \vec{q})-2 \vec{\omega}\right]+\left(\lambda^{\prime}+\eta^{\prime}\right) \nabla(\nabla \cdot \vec{\omega})+\eta^{\prime} \nabla^{2} \vec{\omega} \\
\left\{\rho_{0} C_{V, H}\right\} \frac{D T}{D t}+(1-\varepsilon)\left(\rho_{0} C\right)_{s} \frac{\partial T}{\partial t}=k_{t} \nabla^{2} T+\delta(\nabla \times \vec{\omega}) \cdot \nabla T+Q \\
\rho=\rho_{0}\left[1-\alpha\left(T-T_{a}\right)\right]
\end{gathered}
$$

\subsection{The Steady Solution}

Since the basic state is quiescent

$$
\vec{q}=\vec{q}_{b}=(0,0,0), \quad p=p_{b}(z), \rho=\rho_{b}(z), T=T_{b}(z), \vec{\omega}=\vec{\omega}_{b}(z)=0
$$

The basic state solutions of Equations (3)-(5) and using $T_{b}(0)=T_{0} ; T_{b}(d)=T_{1}$ to be yield

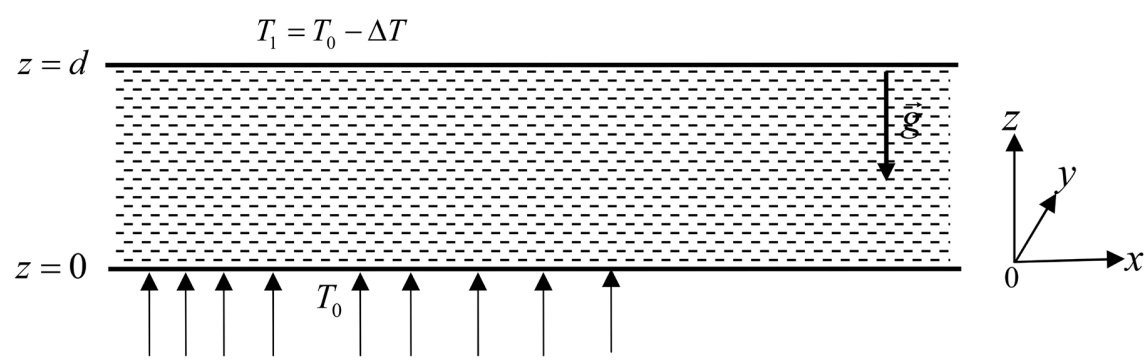

Figure 1. Physical configuration. 


$$
\begin{gathered}
\frac{\partial p_{b}}{\partial z}+\rho_{0} g\left[1+\alpha \frac{Q z^{2}}{2 k_{t}}+\alpha \beta z-\alpha \frac{Q d z}{2 k_{t}}\right]=0, \\
T_{b}(z)=-\frac{Q}{2 k_{t}} z^{2}+\left(\frac{Q d}{2 k_{t}}-\beta\right) z+T_{a}
\end{gathered}
$$

where $\beta=\Delta T / d$ is the temperature gradient. For $Q=0$ in Equation (8), reduced to the linearly decreasing with the distribution in the fluid layer: $T_{b}=-\beta z+T_{a}$.

\subsection{Linear Stability Analysis}

The small perturbation is superimposed on the basic state equations, this gives

$$
(\vec{q}, \rho, p, T)=\left(0, \rho_{b}, p_{b}, T_{b}\right)(z)+(\vec{q}, \rho, p, T)^{\prime}(x, y, z, t)
$$

where primed quantities (') is assumed to be small. Substituting Equation (9) into Equations (2)-(5) using Equations (7) and (8) and linearizing by dropping primes and gives

$$
\begin{gathered}
{\left[\frac{\rho_{0}}{\varepsilon} \frac{\partial}{\partial t}+\frac{1}{k_{t}}(\xi+\eta)\right] \nabla^{2} w=\rho_{0} \alpha g \nabla_{h}^{2} T+2 \xi \nabla^{2} \Omega_{3}} \\
\rho_{0} I \frac{\partial \Omega_{3}}{\partial t}=-2 \xi\left[\frac{1}{\varepsilon} \nabla^{2} w+2 \Omega_{3}\right]+\eta^{\prime} \nabla^{2} \Omega_{3} \\
\rho_{0} C_{1} \frac{\partial T}{\partial t}+\left(w-\delta \Omega_{3}\right)\left[\frac{-Q z}{k_{t}}-\beta+\frac{Q d}{2 k_{t}}\right]=k_{t} \nabla^{2} T
\end{gathered}
$$

where $\rho_{0} C_{1}=\rho_{0} C_{v, H}+(1-\varepsilon) \rho_{s} C_{s}$ and $\nabla_{h}^{2}=\frac{\partial^{2}}{\partial x^{2}}+\frac{\partial^{2}}{\partial y^{2}}$ is the horizontal Laplacian operator. It is now assumed that the perturbation may be stated in expressions of their normal mode kind; thus

$$
\begin{gathered}
w=W(z) \exp \left\{i a_{1} x+i a_{2} x+\sigma t\right\} \\
T=\Theta(z) \exp \left\{i a_{1} x+i a_{2} x+\sigma t\right\} \\
\Omega_{3}=\Omega_{3}(z) \exp \left\{i a_{1} x+i a_{2} x+\sigma t\right\}
\end{gathered}
$$

where $W(z), \Theta(z)$ and $\Omega_{3}(z)$ are the perturbed amplitudes of velocity, temperature and angular velocity respectively. By introducing the non-dimensional variables, we put

$$
(x, y, z)=\left(\frac{x^{*}}{d}, \frac{y^{*}}{d}, \frac{z^{*}}{d}\right), \quad W=\frac{\gamma}{d A} W^{*}, \quad \Theta=\frac{\beta \gamma d}{\kappa} \Theta^{*}, \quad t=\frac{d^{2}}{\gamma} t^{*}
$$

Equations (13)-(15) are substituting into Equations (10)-(12) and then Equation (16) is used to obtain the following stability equations (after dropping the asterisks $\left.{ }^{\star}\right)$ are:

$$
\left\{\frac{1}{D a}\left(D^{2}-a^{2}\right)-\left(D^{2}-a^{2}\right)^{2}\right\} W-a^{2} R_{t} \Theta-2 N_{1}\left(D^{2}-a^{2}\right) \Omega_{3}=0
$$




$$
\begin{aligned}
& \frac{2 N_{1}}{\varepsilon}\left(D^{2}-a^{2}\right) W+\left\{4 N_{1}-N_{3}\left(D^{2}-a^{2}\right)\right\} \Omega_{3}=0 \\
& \left(D^{2}-a^{2}\right) \Theta-[N s(1-2 z)-1]\left[N_{5} \Omega_{3}-W\right]=0
\end{aligned}
$$

The classic value of $M_{2}$ is the order of $10^{-6}$ in different carrier liquids and hence its result is neglected. Equations (17)-(19) are solved the following boundary conditions: on temperature boundary conditions:

1) $\mathrm{RB1}:\left.\Theta\right|_{z=0}=\left.\Theta\right|_{z=1}=0$ for isothermal/isothermal

2) $\mathrm{RB} 2:\left.D \Theta\right|_{z=0}=\left.D \Theta\right|_{z=1}=0$ for insulating/insulating

3) $\mathrm{RB} 3:\left.\mathrm{D} \Theta\right|_{z=0}=\left.\Theta\right|_{z=1}=0$ for insulating/isothermal.

On velocity $W$ and angular velocity $\Omega_{3}$ boundary conditions are:

1) for R-R surface: $W(0,1)=D W(0,1)=\Omega_{3}(0,1)=0$

2) for F-F surface: $W(0,1)=D^{2} W(0,1)=D \Omega_{3}(0,1)=0$

3) lower at rigid surface: $W(0)=D W(0)=\Omega_{3}(0)=0$,

upper at free surface: $W(1)=D^{2} W(1)=D \Omega_{3}(1)=0$.

\section{Method of Solution}

Equations (17)-(19) together with (20) to (23) constitute an eigenvalue $R_{t}$. Thus, the dependent variables are introduced the following base functions:

$$
W=\sum_{i=1}^{N} A_{i} W_{i}(z), \quad \Theta=\sum_{i=1}^{N} B_{i} \Theta_{i}(z), \quad \Omega_{3}=\sum_{i=1}^{N} C_{i} \Omega_{3 i}(z)
$$

Substituting into Equations (16)-(18), then the multiplying on resulting equations respectively by $W_{j}(z), \Theta_{j}(z), \Phi_{j}(z)$ and on integrating, we get

$$
\begin{gathered}
E_{j i} A_{i}+F_{j i} B_{i}+G_{j i} C_{i}=0 \\
H_{j i} A_{i}+I_{j i} B_{i}+J_{j i} C_{i}=0 \\
K_{j i} A_{i}+L_{j i} B_{i}+M_{j i} C_{i}=0
\end{gathered}
$$

where

$$
\begin{gathered}
E_{j i}=\left(1+N_{1}\right)\left\{\left\langle D^{2} W_{j} D^{2} W_{i}\right\rangle+2 a^{2}\left\langle D W_{j} D W_{i}\right\rangle+a^{4}\left\langle W_{j} W_{i}\right\rangle\right\} \\
+D a^{-1}\left(1+N_{1}\right)\left\{\left\langle D W_{j} D W_{i}\right\rangle+a^{2}\left\langle W_{j} W_{i}\right\rangle\right\}, \\
F_{j i}=-a^{2} R_{t}\left\langle W_{j} \Theta_{i}\right\rangle, G_{j i}=-2 N_{1}\left[\left\langle D W_{j} D \Omega_{3 i}\right\rangle+a^{2}\left\langle W_{j} \Omega_{3 i}\right\rangle\right], \\
H_{j i}=\frac{2 N_{1}}{\varepsilon}\left[\left\langle D \Omega_{3 j} D W_{i}\right\rangle+a^{2}\left\langle\Omega_{3 j} W_{i}\right\rangle\right], \quad I_{j i}=0, \\
J_{j i}=-4 N_{1}\left\langle\Omega_{3 j} \Omega_{3 i}\right\rangle-N_{3}\left\langle D \Omega_{3 j} D \Omega_{3 i}\right\rangle+a^{2}\left\langle\Omega_{3 j} \Omega_{3 i}\right\rangle \\
K_{j i}=[N s(1-2 z)-1]\left\langle\Theta_{j} W_{i}\right\rangle, L_{j i}=\left\langle D \Theta_{j} D \Theta_{i}\right\rangle+a^{2}\left\langle\Theta_{j} \Theta_{i}\right\rangle \\
M_{j i}=N_{5}[N s(1-2 z)-1]\left\langle\Theta_{j} \Omega_{3 i}\right\rangle
\end{gathered}
$$


with $\langle\cdots\rangle=\int_{0}^{1}(\cdots) \mathrm{d} z$.

The linear system of Equations (25)-(27) can be reduced to

$$
A X=0 \text {, }
$$

where $A=\left[\begin{array}{ccc}E_{j i} & F_{j i} & G_{j i} \\ H_{j i} & 0 & J_{j i} \\ K_{j i} & L_{j i} & M_{j i}\end{array}\right]$ is the resulting matrix and $X=\left[\begin{array}{c}A_{i} \\ B_{i} \\ C_{i}\end{array}\right]$ is the unknown column matrix.

Equation (28) can have non-trivial solutions if

$$
|A|=0 .
$$

We choose the trial functions of velocity $W_{i}$ and magnetic potential $\Phi_{i}$ satisfying the respective boundary conditions:

1) For R-R surfaces: $W_{i}=\left(z^{i+3}-2 z^{i+2}+z^{i+1}\right), \Omega_{3 i}=\left(z^{i+1}-z^{i}\right)$,

2) For R-F surfaces: $W_{i}=\left(z^{i+3}-5 z^{i+2} / 2+3 z^{i+1} / 2\right), \Omega_{3 i}=\left(z^{i}-z^{i+1} / 2\right)$,

3) For F-F surfaces: $W_{i}=\left(z^{i+3}-2 z^{i+2}+z^{i}\right), \Omega_{3 i}=\left(z^{i-1}\right)$.

On temperature conditions $\Theta_{i}$, we set

$$
\Theta_{i}= \begin{cases}z^{i+1}-z^{i} & \text { for RB1 } \\ z^{i-1} & \text { for RB2 } \\ 1-z^{i+1} & \text { for RB3 }\end{cases}
$$

On substituting we get

$$
f\left(R_{t c}, N_{1}, N_{3}, N_{5}, N s, D a^{-1}, a\right)=0
$$

provides relationships among the related physical parameters $N_{1}, N_{3}, N_{5}, N s, \mathrm{Da}^{-1}$ and thus determines the smallest value of $R_{t}$ corresponding $a_{c}$.

\section{Results and Discussion}

For various physical parameters $N_{1}, N_{3}, N_{5}, N s$ and $D a^{-1}$, the minimum of $R_{t}$ is found with corresponding $a_{c}$. To GT based on WRT is applied to extort the critical eigenvalues. For this purpose, MATHEMATICA 12.0 symbolic algebraic package is applied. The values of $\left(R_{t c}, a_{c}\right)$ computed under the limiting case are compared with Goluskin [24] in Table 1. The results established are in admirable agreement and thus validate the exactness of the numerical technique for in the non-existence of micropolar-porous case $\left(N_{1}=N_{3}=N_{5}=D a^{-1}=N s=0\right)$. Here six convective relationships are concentrated which are three types solely driven by boundary combinations (R-R; solid curve, R-F; dashed curve, F-F; dotted curve) and three types by RB cases (RB1, RB2, RB3) are shown in Figures 2-10.

The neutral stability curves of the system are plotted in Figures 2-5 for different values of $\mathrm{Da}^{-1}, N_{1}, N_{3}$ and $N_{5}$ respectively with three types of ve- 
locity boundary conditions (R-R, R-F, F-F) as well as temperature perturbations (RB1, RB2, RB3). The critical thermal Rayleigh number corresponding to the minimum of each curve increases with $\mathrm{Da}^{-1}$ (Figure 2), $N_{1}$ (Figure 3) and $N_{3}$ (Figure 4); this means that the onset of Rayleigh-Bénard thermal convection becomes more stable on the system. In Figure 5, we observed that the neutral stability curves move downwards for increasing in $M_{5}$ (Figure 5), clearly showing their destabilizing effect on the system.

Table 1. Comparison of $R_{t c}$ and $a_{c}$ for $N_{1}=N_{3}=N_{5}=D a^{-1}=0$.

\begin{tabular}{cccccc}
\hline \multirow{2}{*}{$\begin{array}{c}\text { Temperature } \\
\text { profiles }\end{array}$} & Boundary & \multicolumn{2}{c}{ Goluskin [24] } & \multicolumn{2}{c}{ Present Study } \\
\cline { 3 - 6 } & conditions & $R_{t c}$ & $a_{c}$ & $R_{t c}$ & $a_{c}$ \\
\hline \multirow{2}{*}{ RB1 } & No-Slip & 1707.76 & 3.1163 & 1707.76 & 3.1163 \\
& Free-Slip top & 1100.65 & 2.6823 & 1100.65 & 2.6823 \\
& Free-slip & 657.512 & 2.2214 & 657.512 & 2.2214 \\
\hline \multirow{2}{*}{ RB2 } & No-Slip & 720 & 0 & 720 & 0 \\
& Free-Slip top & 320 & 0 & 320 & 0 \\
& Free-slip & 120 & 0 & 120 & 0 \\
\hline \multirow{2}{*}{ RB3 } & No-Slip & 1295.78 & 2.5519 & 1295.78 & 2.5519 \\
& Free-Slip top & 816.745 & 2.2147 & 816.745 & 2.2147 \\
& Free-slip & 384.693 & 1.7575 & 384.693 & 1.7575 \\
\hline
\end{tabular}

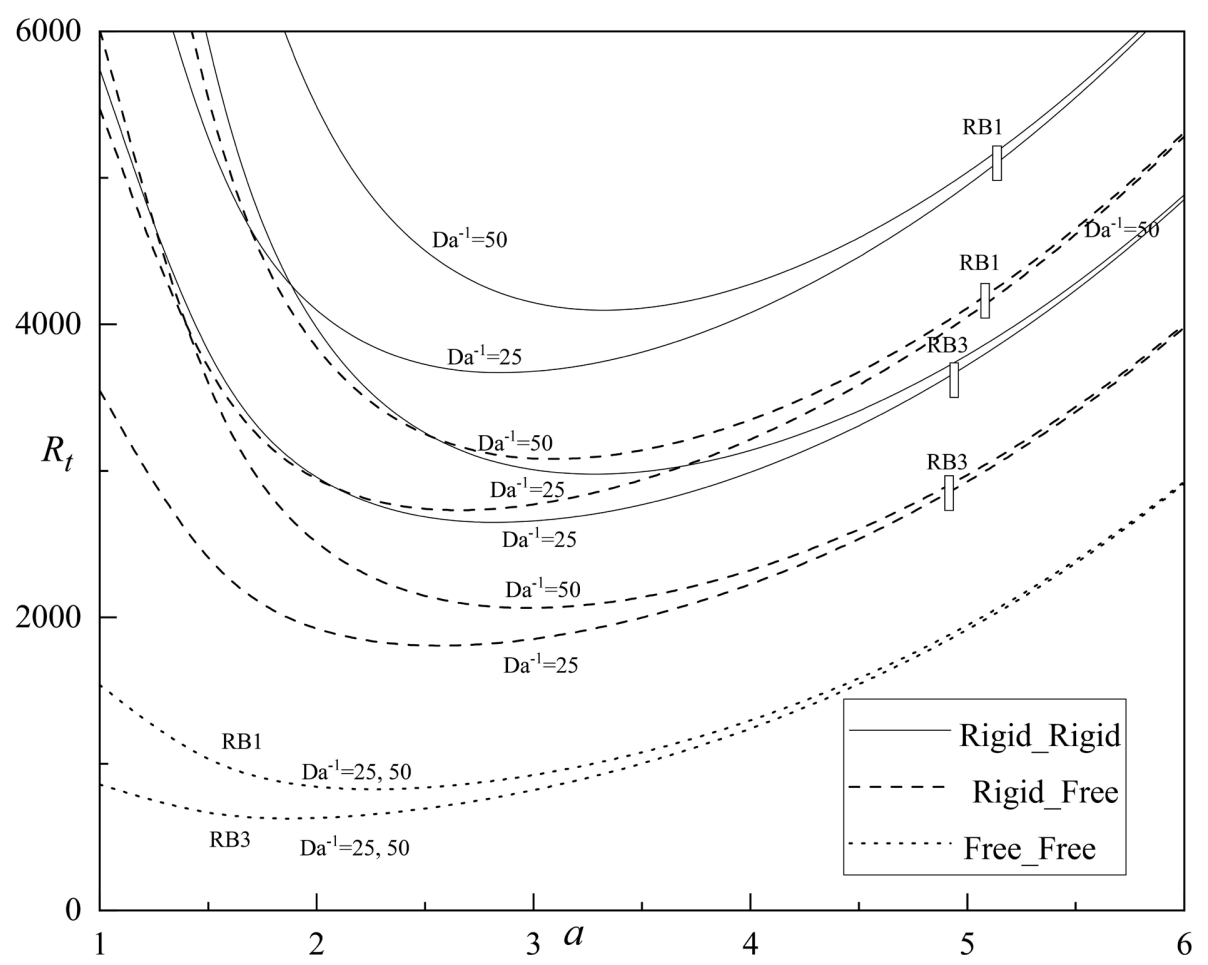

Figure 2. Neutral curves for $N s=N_{3}=2, N_{1}=0.2$ and $N_{5}=0.5$. 


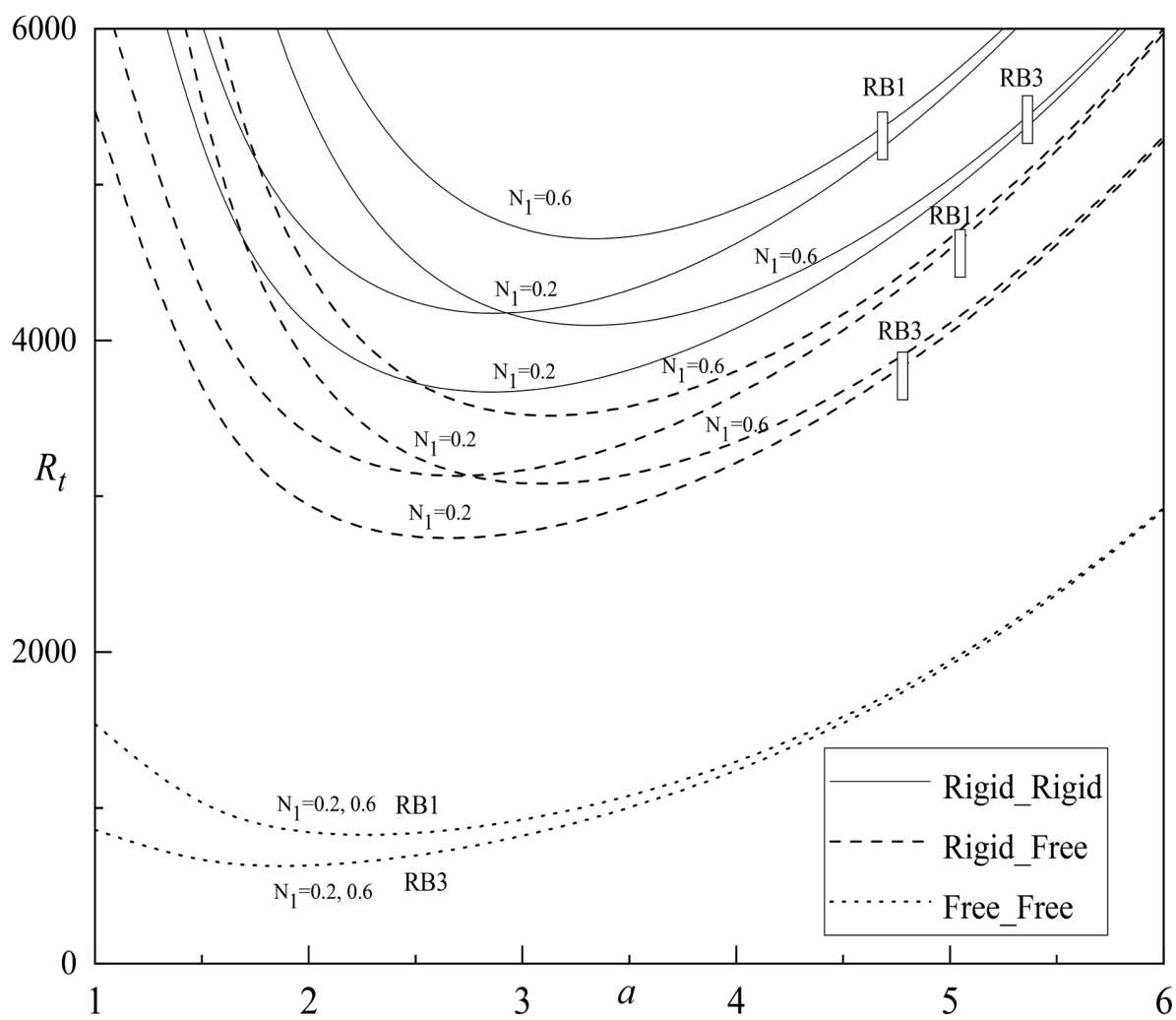

Figure 3. Neutral curves for $N s=N_{3}=2, N_{5}=0.5$ and $D a^{-1}=25$.

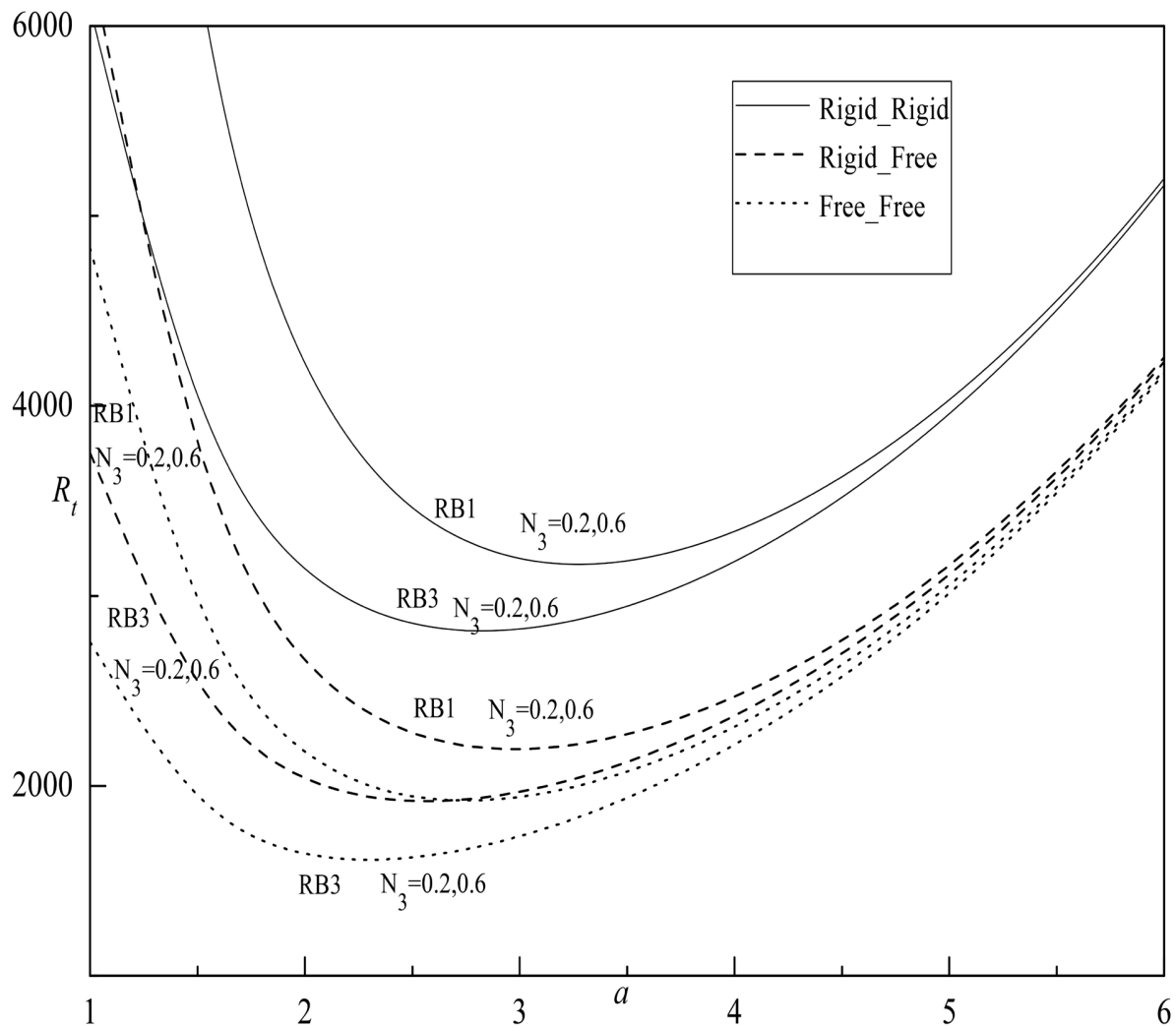

Figure 4. Neutral curves for $N s=2, N_{5}=0.5, N_{1}=0.2$ and $D a^{-1}=25$. 


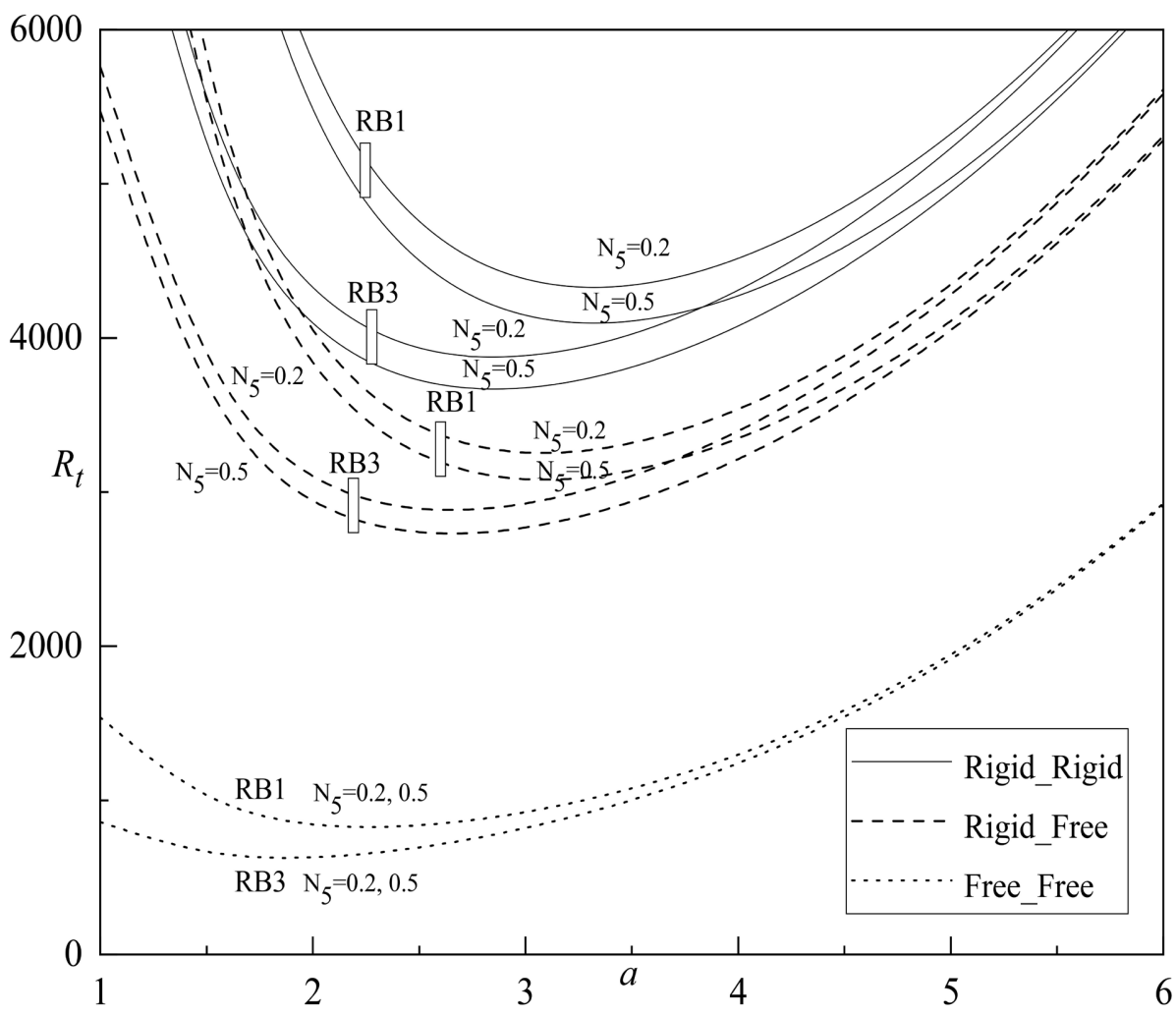

Figure 5. Neutral curves for $N s=N_{3}=2, N_{1}=0.2$ and $D a^{-1}=25$.

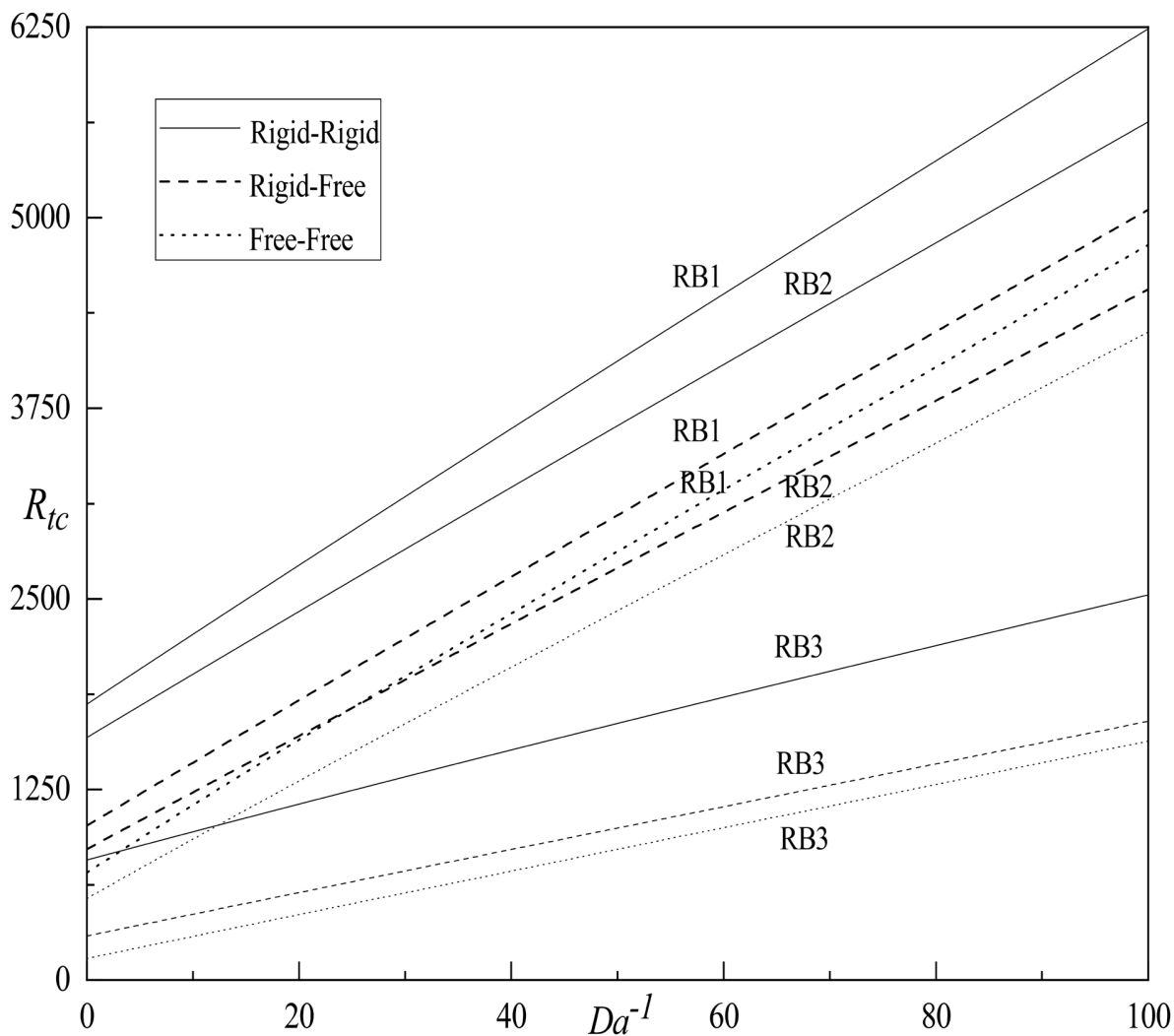

Figure 6. Variation of $R_{t c}$ against $D a^{-1}$ for $N s=N_{3}=2, N_{5}=0.5$ and $N_{1}=0.2$. 


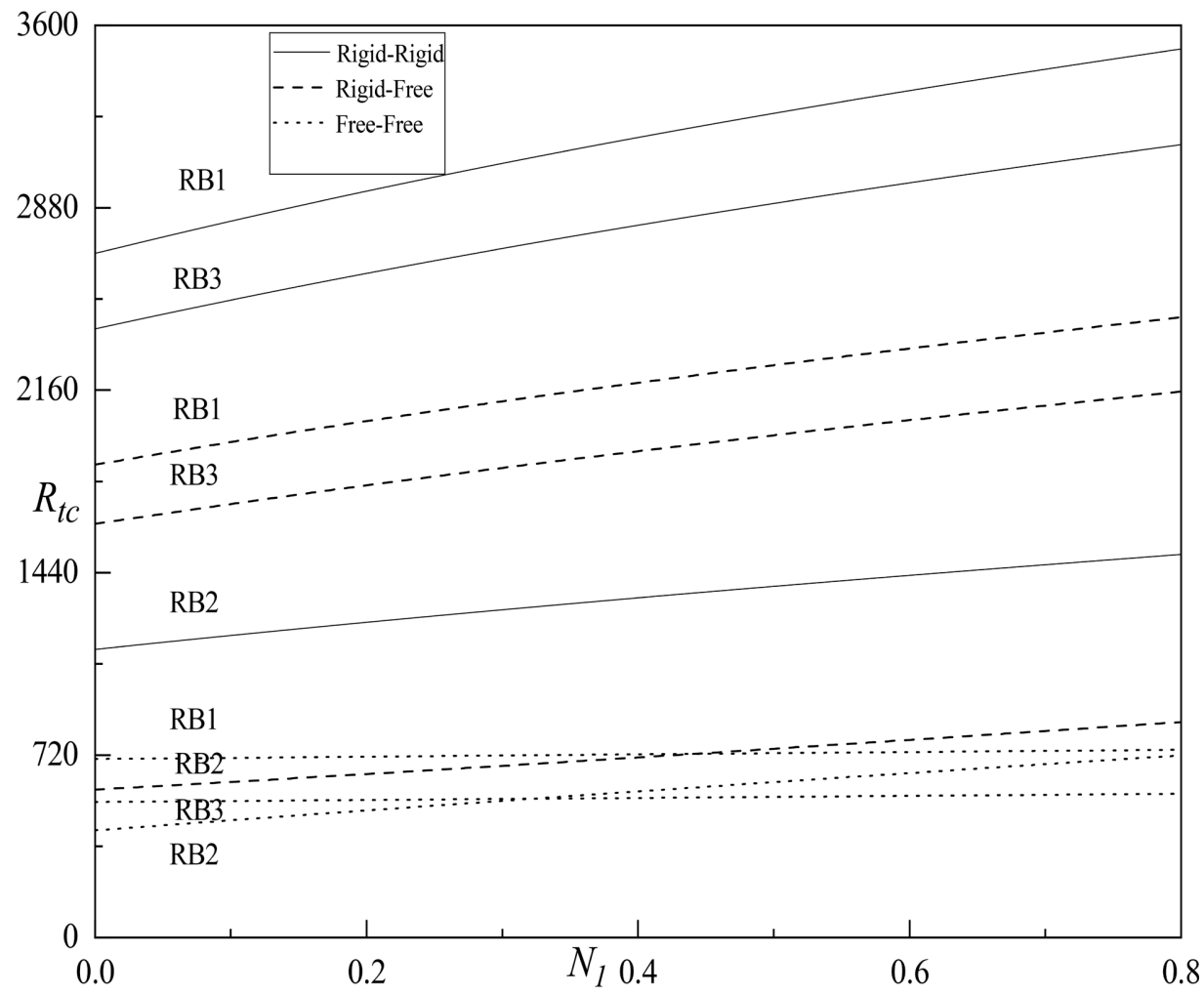

Figure 7. Variation of $R_{t c}$ against $N_{1}$ for $N s=N_{3}=2, N_{5}=0.5$ and $D a^{-1}=25$.

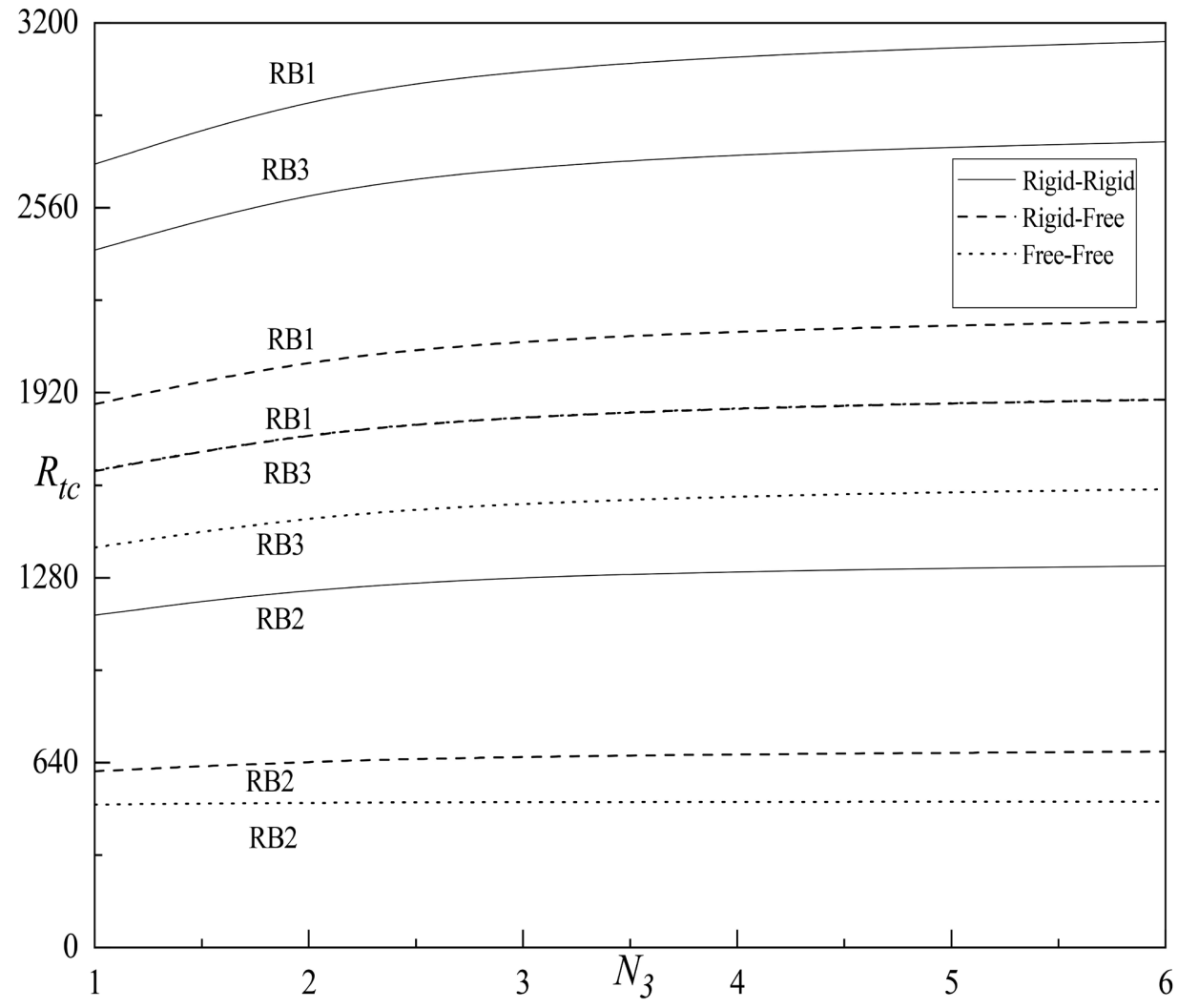

Figure 8. Variation of $R_{t c}$ against $N_{3}$ for $N s=2, D a^{-1}=25, N_{5}=0.5$ and $N_{1}=0.2$. 


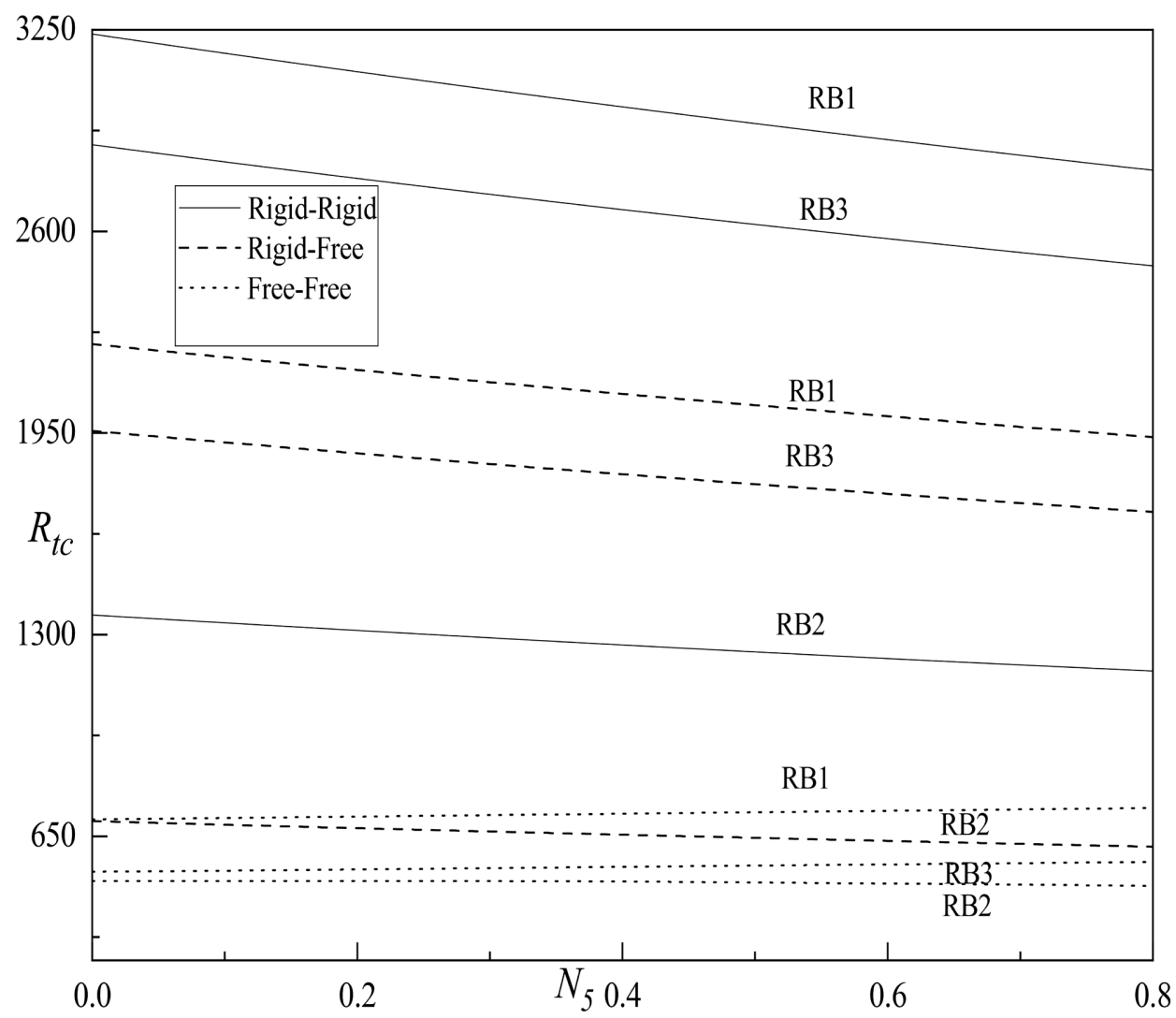

Figure 9. Variation of $R_{t c}$ against $N_{5}$ for $N_{3}=N s=2, D a^{-1}=25$ and $N_{1}=0.2$.

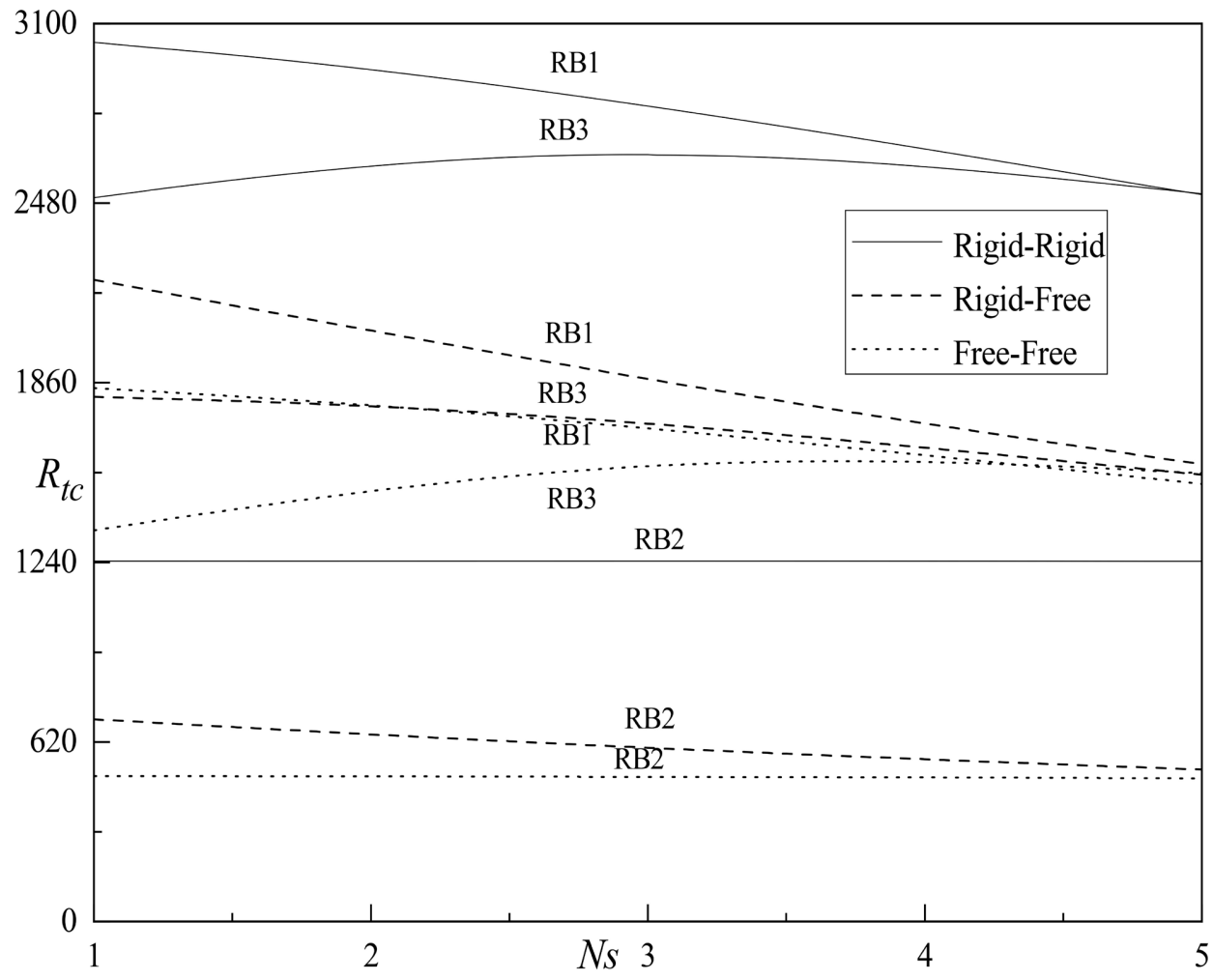

Figure 10. Variation of $R_{t c}$ against $N s$ for $N_{3}=2, D a^{-1}=25, N_{5}=0.5$ and $N_{1}=0.2$. 
Figure 6 represents $R_{t c}$ against $D a^{-1}$ when $N s=N_{3}=2, N_{5}=0.5$ and $N_{1}=0.2$. For a fixed thickness of the porous layer, increase in $D a^{-1}$ amounts to decrease in the permeability of the porous medium which in turn move away the flow of fluid in porous media and hence higher values of $R_{t c}$ is required to the onset of onset of RB porous convection in micropolar fluid layer. Analyzing the $R_{t c}$ with different boundary combinations; bounding surfaces of R-R, RB1 maintaining the highest values of $R_{t c}$ compared with F-F and R-F, RB2 and RB3 surfaces.

In Figure 7, it is observed that $R_{t c}$ increases with increasing $N_{1}$. This is because, as $N_{1}$ increases the concentration of microelements also increases and as a result a greater part of the energy of the system is consumed by these elements in developing gyrational velocities in the fluid which ultimately leads to delay in the onset of micropoalr RB convection. Moreover, the system is found to be more stable when RB1 as compared to the case of RB2 and the system is least stable if the case of RB3.

In Figure 8 plotted $R_{t c}$ as a function of $N_{3}$ for different bounding surfaces of $\mathrm{RB}$ convection when $N s=2, D a^{-1}=25, N_{5}=0.5$ and $N_{1}=0.2$. Here, it is observed that the impact of $N_{3}$ on the stability characteristics of the system is noticeable clearly the critical thermal Rayleigh number $R_{t c}$ increases with increasing $N_{3}$ indicating the spin diffusion (couple stress) parameter $N_{3}$ has a stabilizing effect on the system. This may be attributed to the fact that as $N_{3}$ increases, the couple stress of the fluid increases, which leads to a decrease in microrotation and hence the system becomes more unstable.

Figure 9 shows that increasing the values of the parameter $N_{5}$ with a critical value of the thermal Rayliegh number $R_{t c}$ decreases, hence the onset of micropolar RB convection is destabilized on the system. Nevertheless, the destabilization due to increase in $N_{5}$ is only marginal. In Figure 10 for the system heated from below $N s>0\left(T_{0}>T_{1}\right)$, the internal heat source strength parameter $N s$ increases to make the system more unstable and the results in early the onset of penetrative $\mathrm{RBC}$ in a micropolar ferrofluid saturated porous layer.

\section{Conclusions}

The linear stability theory is used to investigate the onset of penetrative RB convection in a micropolar fluid-saturated porous layer heated from below with different models. The RB convection models are distinguished by the three different temperature boundary combinations like lower and upper at fixed-temperature, lower and upper with fixed-heat flux, or perfectly insulating and bottom surface is fixed-temperature and top surface is fixed-heat flux. The resulting eigenvalue problem is solved numerically by employing the Galerkin type of weighted residual technique. From the foregoing study, the following conclusions may be drawn:

1) The neutral stability curves for various values of physical parameters exhibit that the onset of convection retains its unimodal shape with one distinct 
minimum which defines the critical thermal Rayleigh number and the corresponding wave number.

2) The system is more stabilizing against the convection if the boundaries are $\mathrm{RR}$ with lower and upper at fixed-temperature (RB1) and least stable if the boundaries are $\mathrm{FF}$ with both boundaries insulating to temperature perturbations (RB3).

Thus $\left(R_{\mathrm{tc}}\right)_{\mathrm{FF}}<\left(R_{\mathrm{tc}}\right)_{\mathrm{RF}}<\left(R_{\mathrm{tc}}\right)_{\mathrm{RR}} ;\left(R_{\mathrm{tc}}\right)_{\mathrm{RB} 2}<\left(R_{\mathrm{tc}}\right)_{\mathrm{RB} 3}<\left(R_{\mathrm{tc}}\right)_{\mathrm{RB} 1}$.

3) The effect of increasing the value of coupling parameter $N_{1}$ and spin diffusion (couple stress) parameter $N_{3}$ is to delay, while increasing the Darcy number $D a$, micropolar heat conduction parameter $N_{5}$ and internal heating Ns is to hasten the onset of $\mathrm{RB}$ convection.

4) The effect of increasing $\mathrm{Da}^{-1}, N_{1}$ as well as decrease in $N_{5}$ is to increase the critical wave number.

5) The value of $a_{c}$ in RB1 case is always found to be higher than in RB3 case. That is $\left(a_{c}\right)_{\mathrm{RB} 3}<\left(a_{c}\right)_{\mathrm{RB} 1}$.

For future study, it is intended to extend this work to the Rayleigh-Benard convection in a micropolar fluid saturated porous layer in the presence of vertical channel. The eigenvalue problem is solved by Chebyshev collocation method. This will allow investigating natural and mixed convection in vertical fluid layer.

\section{Conflicts of Interest}

The authors declare no conflicts of interest regarding the publication of this paper.

\section{References}

[1] Eringen, A.C. (1980) Theory of Anisotropic Micropolar Fluids. International Journal of Engineering Science, 18, 5-17. https://doi.org/10.1016/0020-7225(80)90003-8

[2] Eringen, A.C. (1978) Micropolar Theory of Liquid Crystals. In: Johnson, J.F. and Porter, R.S., Eds., Liquid Crystals and Ordered Fluids, Vol. 3, Plenum, New York, 443-474. https://doi.org/10.1007/978-1-4615-8888-7 30

[3] Lebon, G. and Perez-Garcia, C. (1981) Convective Instability of a Micropolar Fluid Layer by the Method of Energy. International Journal of Engineering Science, 19, 1321-1329. https://doi.org/10.1016/0020-7225(81)90015-X

[4] Payne, L.E. and Straughan, B. (1989) Critical Rayleigh Numbers for Oscillatory and Non-Linear Convection in an Isotropic Thermomicropolar. International Journal of Engineering Sciences, 27, 827-836. https://doi.org/10.1016/0020-7225(89)90048-7

[5] Idris, R., Othman, H. and Hashim, I. (2009) On Effect of Non-Uniform Basic Temperature Gradient on Bénard-Marangoni Convection in Micropolar Fluid. International Communications in Heat and Mass Transfer, 36, 255-258. https://doi.org/10.1016/j.icheatmasstransfer.2008.11.009

[6] Mahmud, M.N., Mustafa, Z. and Hashim, I. (2010) Effects of Control on the Onset of Bénard-Marangoni Convection in a Micropolar Fluid. International Communications in Heat and Mass Transfer, 37, 1335-1339. https://doi.org/10.1016/j.icheatmasstransfer.2010.08.013

[7] Sharma, R.C. and Kumar, P. (1995) On Micropolar Fluids Heated from Below in 
Hydromagnetics. Journal of Non-Equilibrium Thermodynamics, 20, 150-159. https://doi.org/10.1515/jnet.1995.20.2.150

[8] Zahn, M. and Greer, D.R. (1995) Ferrohydrodynamics Pumping in Spatially Uniform Sinusoidally Time Varying Magnetic Fields. Journal of Magnetism and Magnetic Materials, 149, 165-173. https://doi.org/10.1016/0304-8853(95)00363-0

[9] Rudraiah, N., Shivakumara, I.S. and Nanjundappa, C.E. (1998) Effect of Non-Uniform Concentration Distribution on Double Diffusive Convection in Magnetic Fluids. Indian Journal of Engineering \& Materials Sciences, 5, 427-435. http://nopr.niscair.res.in/handle/123456789/29668

[10] Abraham, A., (2002) Rayleigh-Bénard Convection in a Micropolar Magnetic Fluids. International Journal of Engineering Science, 40, 449-460. https://doi.org/10.1016/S0020-7225(01)00046-5

[11] Sunil, Chand, P., Bharti, P.K. and Mahajan, A. (2008) Thermal Convection in Micropolar Ferrofluid in the Presence of Rotation. Journal of Magnetism and Magnetic Materials, 320, 316-324. https://doi.org/10.1016/j.jmmm.2007.06.006

[12] Nanjundappa, C.E., Shivakumara, I.S. and Srikumar, K. (2013) The Onset of Ferromagnetic Convection in a Micropolar Ferromagnetic Fluid Layer Heated from Below. Journal of Electromagnetic Analysis and Applications, 5, 120-133. https://doi.org/10.4236/jemaa.2013.53020

[13] Rudraiah, N. and Sekhar, G.N. (1991) Convection in Magnetic Fluids with Internal Heat Generation. ASME Journal of Heat Transfer, 113, 122-127.

https://doi.org/10.1115/1.2910514

[14] Shivakumara, I.S., Rudraiah, N. and Nanjundappa, C.E. (2002) Effect of Non-Uniform Basic Temperature Gradient on Rayleigh-Bénard-Marangoni Convection in Ferrofluids. Journal of Magnetism and Magnetic Materials, 248, 379-395.

https://doi.org/10.1016/S0304-8853(02)00151-8

[15] Shivakumara, I.S. and Nanjundappa, C.E. (2006) Marangoni Ferroconvection with Different Initial Temperature Gradients. International Journal of Heat and Mass Transfer, 28, 45-60.

[16] Shivakumara, I.S. and Nanjundappa, C.E. (2006) Effects of Coriolis Force and Different Basic Temperature Gradients on Marangoni Ferroconvection. Acta Mechanica, 182, 113-124. https://doi.org/10.1007/s00707-005-0296-1

[17] Singh, J. and Bajaj, R. (2011) Convective Instability in a Ferrofluid Layer with Temperature-Modulated Rigid Boundaries Fluid. Fluid Dynamics Research, 43, Article ID: 025502. https://doi.org/10.1088/0169-5983/43/2/025502

[18] Nanjundappa, C.E., Shivakumara, I.S., Lee, J. and Ravisha, M. (2011) Effect of Internal Heat Generation on the Onset of Brinkman-Benard Convection in a Ferrofluid Saturated Porous Layer. International Journal of Thermal Sciences, 50, 160-168. https://doi.org/10.1016/j.ijthermalsci.2010.10.003

[19] Nanjundappa, C.E., Shivakumara, I.S. and Prakash, H.N. (2012) Penetrative Ferroconvection via Internal Heating in a Saturated Porous Layer with Constant Heat Flux at the Lower Boundary. Journal of Magnetism and Magnetic Materials, 324, 1670-1678. https://doi.org/10.1016/j.jmmm.2011.11.057

[20] Nanjundappa, C.E., Shivakumara, I.S. and Srikumara, K. (2013) On the Penetrative Bénard Marangoni Convection in a Ferromagnetic Fluid Layer. Aerospace Science and Technology, 27, 57-66. https://doi.org/10.1016/j.ast.2012.06.007

[21] Rômulo Santos, D.C., Sílvio Gama, M.A. and Ramiro Camacho, G.R. (2018) Two-Dimensional Simulation of the Navier-Stokes Equations for Laminar and Turbulent Flow around a Heated Square Cylinder with Forced Convection. Applied 
Mathematics, 9, 291-312. https://doi.org/10.4236/am.2018.93023

[22] Rashad, M.A., Khan, A.W., EL-Kabeir, M.M.S. and EL-Hakiem, M.A.A. (2019) Mixed Convective Flow of Micropolar Nanofluid across a Horizontal Cylinder in Saturated Porous Medium. Applied Sciences, 9, Article 5241.

https://doi.org/10.3390/app9235241

[23] Islam, M.R., Nasrin, S. and Alam, Md.M. (2020) Unsteady Electromagnetic Free Convection Micropolar Fluid Flow through a Porous Medium along a Vertical Porous Plate. Open Journal of Applied Sciences, 10, 701-718. https://doi.org/10.4236/ojapps.2020.1011049

[24] Goluskin, D. (2015) Internally Heated Convection and Rayleigh-Bénard Convection. Springer, Berlin. https://doi.org/10.1007/978-3-319-23941-5 


\section{Nomenclature}

\section{List of Symbols}

$a=\left(a_{1}^{2}+a_{2}^{2}\right)^{1 / 2}$ horizontal wave number

$C_{V, H}$ specific heat at constant volume and magnetic field

$D=\mathrm{d} / \mathrm{d} z$ differential operator

$D a=k / d^{2}$ Darcy number

$D / D t=\partial / \partial t+\vec{q} \cdot \nabla \quad$ convective derivative

$f(z)$ internal heat source strength

$\vec{g}$ acceleration due to gravity

Imoment of inertia

$k_{t}$ thermal conductivity

$K_{2}$ pyromagnetic co-efficient

$K=6 \pi \tilde{\mu} \eta$

$N_{1}=\xi_{r} / \eta$ coupling parameter

$N_{3}=\eta^{\prime} / \eta d^{2}$ spin diffusion (couple stress) parameter

$N_{5}=\delta / \rho_{0} C_{0} d^{2}$ micropolar heat conduction parameter

$N s=Q d / 2 \beta k_{1}$ internal heat source strength

$p$ pressure

$P_{r}$ Prandtl number

$Q$ internal heat source strength

$R_{t}=\alpha \beta g d^{4} / v \kappa$ thermal Rayleigh number

$\vec{q}$ velocity

$T$ temperature

Ta Average temperature

\section{Greek Symbols}

$\alpha$ coefficient of thermal expansion

$\beta$ uniform temperature gradient

$\xi$ vortex viscosity

$\omega$ micro rotation

$\alpha$ coefficient of thermal expansion

$\delta$ micropolar heat conduction coefficient

$\nabla=\frac{\partial}{\partial x}+\frac{\partial}{\partial y}+\frac{\partial}{\partial z}$

$\nabla^{2}=\partial^{2} / \partial x^{2}+\partial^{2} / \partial y^{2}+\partial^{2} / \partial z^{2}$

$\nabla_{h}^{2}=\frac{\partial^{2}}{\partial x^{2}}+\frac{\partial^{2}}{\partial y^{2}}$

$\phi$ magnetic potential

$\chi$ magnetic susceptibility

$\Lambda$ ratio of viscosity

$\eta$ shear kinematic viscosity co-efficient

$\eta^{\prime}$ shear spin viscosity co-efficient 
$\lambda^{\prime}$ bulk spin viscosity coefficient

$\mu_{0}$ free space magnetic permeability

$\mu_{f}$ dynamic viscosity

$\tilde{\mu}_{f}$ effective viscosity

$v$ kinematic viscosity of a fluid

$\rho$ density

$\rho_{0}$ mean density of the clean fluid

$\theta$ perturbation in temperature

\section{Subscripts}

b Basic state

f Fluid

0 Reference quantities 